\title{
THE ORIENTAL LEPIDOSTOMATIDAE (TRICHOPTERA) DESCRIBED BY BANKS AND HAGEN ${ }^{1}$
}

\author{
By JoHn S. WeAver III \\ The Department of Entomology \\ The University of New Hampshire \\ Durham, NH, 03824, USA.
}

\section{INTRODUCTION}

There are 14 Oriental and 1 eastern Palearctic (Japan) species of Lepidostomatidae described by Banks and Hagen (Fischer 1970). Unfortunately, the original descriptions of these species are not very useful for identification purposes today, because neither author relaxed and cleared the genitalia of these species. This technique reveals important characters of caddisfly genitalia and is now a standard method in Trichopterology. Hence, the redescription of these species is a desired prerequisite for further contributions to the Oriental lepidostomatids. Fortunately, syntypes of all these species have been located; most were at the Museum of Comparative Zoology (MCZ), Harvard University, Massachusetts and a few were at the British Museum (Natural History), London.

Banks and Hagen rarely indicated where all syntypes of their new species were deposited. Judging from the collection of Trichoptera at the MCZ, I infer they exchanged specimens, including syntypes, with their colleagues. Most of their syntypes are at the MCZ, but many are also in several other museums. For example, Ulmer (1951) mentioned that a few lepidostomatid types of Banks were in the "Museum Selangor" (probably the National Museum of Malaysia, Kuala Lumpur) and for this work I found male syntypes of two species only at the British Museum.

Also, Banks and Hagen did not label unique species types as holotypes. Banks' Trichoptera syntypes at the MCZ, that I have examined, have red type labels of two varieties. The first and older kind of label is glossy red obverse and white reverse, reading "Type"

\footnotetext{
'Scientific Contribution Number 1350 from the New Hampshire Agriculture Experiment Station.

Manuscript received by the editor April 9, 1985.
} 
on the first line and with a species specific number on the second line. All of Hagen's syntypes examined bear MCZ labels of this variety. The second kind of type label is on nonglossy red paper, reading "M.C.Z." on the first line, "Type" or "Paratype" on the second line and with a species specific number on the third line. Also, the first syntype of the series with the red label reading "Type" has an additional label of determination that has "type" written in the lower left corner; other syntypes have red labels reading "Paratype," but usually not have larger determination labels. The change in type labels of Banks' species took place sometime after MCZ type no. 16500 of Goerodes grandis (Banks 1931c), but before type no. 20182 of Goerodes medius (Banks 1934).

Lectotypes have been designated for all species examined, even when only one syntype was available, because of the possibility that additional syntypes might exist at other institutions. For each species I have selected as lectotype the first specimen in the type series which bears a separate determination label. Ross (1938) described the important details of Hagen's and Banks' type labels and I have followed his procedure for selecting lectotypes, which was recommended to him by Banks.

In this work, the bibliography following each species lists selected references. In the examined material cited, the institutions or persons holding the specimens are indicated in parentheses and are abbreviated as follows: British Museum (Natural History), BMNH; Canadian National Collection, CNC; Museum of Comparative Zoology, MCZ; Smithsonian Institution, National Museum of Natural History, USNM; and the collections of Hans Malicky, HM; and the author, JSW. Also, known collectors are abbreviated as follows: Baumann and Cross, B\&C; C. S. Clagg, CSC; Davis and Rowe, D\&R; O. S. Flint, Jr., OSF; M. R. Henderson, MRH; K. V. Krombein, KVK; and H. M. Pendlebury, HMP.

\section{LEPIDOSTOMATIDAE}

\section{Goerodes abruptus Banks}

(Fig. 1)

Goerodes abrupta Banks 1931b: 391, ㅇ, fig. 40.

Goerodes abruptus, Fischer 1970: 17.

Male. Unknown.

Female. Wings brown, fore wing $6.2 \mathrm{~mm}$ long. Genitalia (Fig. 
1), spermathecal sclerite trapezoidal with posterolateral corners extended, in ventral view.

Material Examined. Lectotype $^{\text {, }}$ "Type 16473," MALAYSIA, PERAK, Batang Padang, Jor Camp, 1800 ft, 10 III 1924, HMP, (MCZ).

\section{Goerodes apoanus (Banks)}

(Figs. 2, 3, 4)

Goerinella apoana Banks 1937: 155, ð̊ㅇ, pl. 5 figs. 49, 51.

Goerodes apoanus, Fischer 1970: 17.

Male. Scape $1.2 \mathrm{~mm}$ long, not highly modified, cylindrical, mesal surface concave. Maxillary palp $1.0 \mathrm{~mm}$ long, similar as in $G$. conjunctus (Fig. 6B). Fore wing (Fig. 2) $8.7 \mathrm{~mm}$ long, modified with narrow anterior costal fold. Genitalia (Fig. 3).

Female. Genitalia (Fig. 4); spermatheca ovoid, microtrichia with a conspicuous pattern, short and dense in anterior $1 / 3$, fewer and larger in middle 1/3, sparse and short in posterior $1 / 3$.

Material Examined. PHILIPPINES, MINDANAO: Lectotype $\hat{\jmath}$, “M.C.Z. Type 22049," Mt. Apo, 6000 ft, Mainit Riv., 22 IX, CSC, (MCZ). Paratypes, "M.C.Z. Paratype 22049:" same data as lecto-

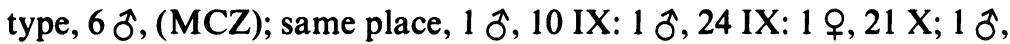
23 X, (MCZ); Mt. Apo, Galog Riv., 6000 ft, 1 क, 1 ڤิ, 26 IX; 1 ô, 18 X, 2 ô, 1 ㅇ, 19 X; 1 ô, 21 X; 1 ㅇ, 6 XI, CSC, (MCZ); Mt. Apo., Sibulan Riv., 7-8000 ft, 1 ô, 6 IX, CSC, (MCZ). Nontypes: Mt. Apo., Galog Riv., $6000 \mathrm{ft}, 1$ \%, 1 IX; 1 ㅇ, 8 X; 1 ㅇ, 18 X; 3 ㅇ, 19 X; 1 ô, 21 X; 1 ᄋ, XI; 1 đิ, 4 XI, CSC, (MCZ); Mt. Apo, Mainit Riv., $6000 \mathrm{ft}, 1$ \&, 22 IX, CSC, (MCZ); Mt. Apo, Sibulan Riv., 7-8000 ft, 1 ô, 1 ᄋ, 6 IX, CSC, (MCZ); Mt. Mayo, Davao, 4-5000 ft. 1 \%, 26 I; 1 ㅇ, 30 I, CSC, (MCZ).

\section{Goerodes bicolor (Banks)}

(Fig. 5)

Goerinella bicolor Banks 1937: 155-6, \&, pl. 5 fig. 45.

Goerodes bicolor, Fischer 1970: 18.

Male. Unknown.

Female. Scape $1.7 \mathrm{~mm}$ long, cylindrical. Fore wing brown, 12.0 $\mathrm{mm}$ long. Eyes dark brown, rest of body orangish brown. Genitalia (Fig. 5), spermatheca with a few microtrichia, lacking any conspicuous pattern.

Material Examined. Lectotype ㅇ, “M.C.Z. Type 22050," PHILIPPINES, MINDANAO, La Lun Mts., Davao, 4 VII, CSC, (MCZ). 


\section{Goerodes conjunctus (Banks)}

(Figs. 6, 7, 8)

Goerinella conjuncta Banks 1934: 574-5, ठ̊̊o, figs. 10, 20.

Goerodes conjunctus, Fischer 1970: 18-9.

Male. Color brown. Scape (Fig. 6B) $1.7 \mathrm{~mm}$ long, mesal surface concave with modified setae and lobes. Maxillary palps (Fig. 6A), basal segment curved, apical segment flexible and setose. Fore wing (Fig. 6C) $9.2 \mathrm{~mm}$ long. Genitalia (Fig. 7).

Female. Scape $1.2 \mathrm{~mm}$ long. Fore wing $8.6 \mathrm{~mm}$ long. Genitalia (Fig. 8).

Material Examined. Lectotype ô: MALAYSIA, SABAH, Kinabalu, Lumu Lumu, $5000 \mathrm{ft}, 16 \mathrm{IV},($ BMNH); "Allotype," Lumu Lumu, $5500 \mathrm{ft}, 1$ ㅇ, 16 IV 1929, (BMNH).

\section{Goerodes grandis (Banks)}

(Figs. 9, 10, 11)

Goerinella grandis Banks 1931c: 420, ठิ

Goerodes grandis, Fischer 1970: 20-1.

Male. Color brown. Scape (Fig. 9B) $2.0 \mathrm{~mm}$ long, with mesal surface modified. Maxillary palp $2.0 \mathrm{~mm}$ long, similar as in $G$. conjunctus (Fig. 6B). Fore wing (Fig. 9A) $13.5 \mathrm{~mm}$ long. Genitalia (Fig. 10).

Female. Color brown. Scape $1.5 \mathrm{~mm}$ long. Fore wing $14.0 \mathrm{~mm}$ long. Genitalia (Fig. 11).

Material Examined. Malaysia, SABAH: Lectotype $\hat{0}$, "Type 1650," Pakka, Mt. Kinabalu, 10000 ft, 23 III 1929, HMP, (MCZ); “CNC No. 12359 Paratype," same place: 1 đo, 20 III 1929, HMP, (CNC); 1 \&, 23 III 1929, HMP, (MCZ); 2 ㅇ, 22 III 1929, HMP, (MCZ).

\section{Goerodes medius (Banks)}

(Figs. 12, 13, 14)

Goerinella media Banks 1934: 574-5, ðิo ㅇ, figs. 11, 17, 18, 22.

Goerodes medius, Fischer 1970: 21-2.

Male. Color brown. Scape $0.9 \mathrm{~mm}$ long, not highly modified. Maxillary palp $0.5 \mathrm{~mm}$ long, curved in front of frons. Fore wing (Fig. 12) $8.0 \mathrm{~mm}$ long. Genitalia (Fig. 13).

Female. Scape $1.0 \mathrm{~mm}$ long. Fore wing $6.5-7.5 \mathrm{~mm}$ long. Genitalia (Fig. 14). 
Material Examined. MALAYSIA, SABAH: Lectotype ô, Mt. Kinabalu, Lumu Lumu, $5500 \mathrm{ft}, 10 \mathrm{IV}, \mathrm{HMP},(\mathrm{BMNH})$; "Allotype," same place, 1 \%, 14 IV 1929, HMP, (BMNH); "M.C.Z. Type 20183," "Paratype," same place, but $7000 \mathrm{ft}, 1$ \% $/$ o head and abdomen, 3 IV 1929, HMP, (MCZ); same place, but 5500 ft, 1 \%, 17 IV 1929, HMP, (BMNH); same data, 1 \%, (MCZ).

\section{Goerodes minor (Banks)}

(Figs. 15, 16)

Goerinella minor Banks 1931a: 67, ô, pl. 5 fig. 6.

Goerodes minor, Fischer 1970: 22.

Male. Color brown. Scape $0.9 \mathrm{~mm}$ long, cylindrical, not highly modified. Maxillary palp $0.5 \mathrm{~mm}$ long, curved in front of frons, similar as in G. conjunctus (Fig. 6B). Fore wing (Fig. 15) $8.0 \mathrm{~mm}$ long. Genitalia (Fig. 16).

Female. Unknown.

Material Examined. Lectotype ô, "Type 16418," PHILIPPINES, LUZON, Mt. Mekiling, no date, Baker, (MCZ).

\section{Goerodes piscinus (Hagen)}

(Fig. 17)

Mormonia piscina Hagen 1859: 208.

Goerodes piscina, Mosely 1949: 784-5, ô 우, figs. 167-71.

Goerodes piscinus, Fischer 1970: 23.

Male. Color brown, cf. (Mosely 1949: figs. 167-71). Scape 1.3 $\mathrm{mm}$ long, not highly modified. Maxillary palp $0.8 \mathrm{~mm}$ long.

Female. Scape $1.2 \mathrm{~mm}$ long. Fore wing $9.1 \mathrm{~mm}$ long. Genitalia (Fig. 17).

Material Examined. SRI LANKA: Lectotype ô, "Type 10946," no date, (MCZ); 1 ô syntype, (MCZ); 1 क (Hagen and Mosely det. as ursina) with BMNH type label, (BMNH); N. E. DIST.: Kanda-ela, Resevior, $5.6 \mathrm{mi}$. SW Nuwara Eliya, $6200 \mathrm{ft}, 5 \hat{\jmath}, 1$ ㅇ, 10-21 II 1970, D\&R, (USNM); 2 ô, 1 ᄋ' 1-5 X 1970, OSF, (USNM); 1 ô, 31 V-2 VI 1976, KVK et al., (USNM); Great Western Est. nr Talawalele, $4200 \mathrm{ft}, 1$ ठै, 1 \&, 5 X 1970, OSF, (USNM); Pattipola, 1 ô, V 1911, J. C. Fryer, (BMNH); Pattipola, $6100 \mathrm{ft}, 2$ o, 2 o, 3-6 X 1970, OSF, (USNM); Horton Plains, Agrapatana Rd., 6600.ft, 5 ô, 4 \&, $4 \mathrm{X}$ 1970, OSF, (USNM); same place, but $7000 \mathrm{ft}, 1 \hat{\delta}, 20$ III 1973, B\&C, (USNM); same place, but $6000 \mathrm{ft}, 1$ ठิ, 2 \&, 21 III 1973, B\&C, 
(USNM); Lovers Leap Creek, $7000 \mathrm{ft}, 5 \hat{\jmath}, 15$ III 1973, B\&C, (USNM); Moon Plains, $1635 \mathrm{ft}, 11$ ô, 13 ᄋ, 18 VIII 1973, Ginter Ekis, (USNM); Nuwara Eliya, $1 \hat{\jmath}, 25$ VII 1924, (BMNH); $2 \hat{\jmath}, 1$ ㅇ, 24 IX 1975; 1 ô, 2 ㅇ, 26-27 IX 1975, D. M. Davis et al., (USNM); 1 ô, 27-29 V 1975, D. H. Messersmith et al., (USNM); Nuwara Eliya, Galway Reserve, 2 \%, 28 IX-1 X 1973, KVK et al., (USNM); Hakgala, Botanic Garden, Circuit Bung., 1 ô, 1 क, 5-8 II 1979, KVK et al., (USNM); Botanical Gardens, 6000 ft, 3 \&, 6-8 X 1976, G. F. Hevel et al., (USNM).

\section{Goerodes posticatus (Banks)}

(Figs. 18, 19)

Goerinella posticata Banks 1931b: 393, ఫૈ, figs. 35, 37, 38.

Goerodes posticatus, Fischer 1970: 23.

Male. Color brown. Scape $1.3 \mathrm{~mm}$ long, with basomesal lobe. Maxillary palp $1.2 \mathrm{~mm}$ long. Fore wing (Fig. 18) $7.7 \mathrm{~mm}$ long, with long posterior marginal fold. Genitalia (Fig. 19).

Female. Unknown.

Material Examined. MALAysia: Lectotype ô, "Type 16471," PAHANG, Cameron's Highlands, Tahan Padang, $4500 \mathrm{ft}, 13$ VI 1924, MRH, (MCZ); Cameron's Highlands, 4-5000 ft, 1 ô, 10 VI 1935, HMP, (MCZ); SELANGOR, Bnkit Kutu, 3500 ft, 1 ô, 14 III 1931, HMP, (MCZ).

\section{Goerodes ursinus (Hagen)}

(Fig. 20)

Mormonia ursina Hagen 1858: 484.

Goerodes ursina Mosely 1949: 782-3, ㅇ, fig. 166.

Goerodes punda Mosely 1949: 785, $\widehat{\sigma}$, figs. 172-7, NEW SYNONYM.

Goerodes punda, Fischer 1970: 23.

Goerodes ursinus, Fischer 1970: 25.

Male. Color brown, cf. (Mosely 1949: figs. 172-7). Scape 1.1 $\mathrm{mm}$ long, not highly modified. Maxillary palps $0.7 \mathrm{~mm}$ long. Fore wing 6.0-7.4 mm long.

Female. Scape $1.0 \mathrm{~mm}$ long. Fore wing $7.0-8.5 \mathrm{~mm}$ long. Genitalia (Fig. 20). Spermatheca ovoid, microtrichia in anterior $1 / 4$ dense and stout, having reticulate pattern, in middle $1 / 2$ long, in posterior $1 / 4$ short and sparse.

Material Examined. SRI LANKA: Lectotype $ᄋ$, "Type 10943," (MCZ); 1 \& syntype, no date, (MCZ); 1 \%, (MCZ); BAD. DIST.: 
Koslanda, Diyaluma Falls, $3000 \mathrm{ft}, 6$ ô, 7 ㅇ, 19 III 1973, B\&C, (USNM); COL. DIST.: Pudukka, $300 \mathrm{ft}, 1$,, 16 XI 1970, OSF, (USNM); Tunmodera, $200 \mathrm{ft}, 2$ $\widehat{0}, 17$ XI 1970, OSF, (USNM); GAL. DIST., Sinharaja Jungle, Kanneliya sec., 1 ô, 13-16 July 1978, KVK et al, (USNM); KAN. DIST.: Polpitiya, Kelani Ganga, $400 \mathrm{ft}, 1$ ठิ, 24 IX 1970, OSF, (USNM): Laksapana, $1200 \mathrm{ft}, 1$ \%, 23-29 IX 1970, OSF, (USNM); MATE. DIST.: Elkaduwa, Hunas Falls, $3000 \mathrm{ft}, 6$ ठิ, 6 O, 5 IV 1973, B\&C, (USNM); RAT. DIST.: Belihul Oya, $2000 \mathrm{ft}, 1$ ô, 12 X 1970, OSF, (USNM); same place, 3 tิ, 2 ㅇ, 22 III 1973, B\&C, (USNM); Uggalkaltota, $350 \mathrm{ft}$, irrigation bungalow, 1 ⿵人, 21 I-8 II 1970, D\&R, (USNM); Uggalkaltota, 10ิ, 23-26 VI 1978, KVK et al., (USNM).

\section{Goerodes venularis (Banks)}

(Figs. 21, 22, 23)

Goerinella venularis Banks 1931b: 392, ठิ figs. 34, 36.

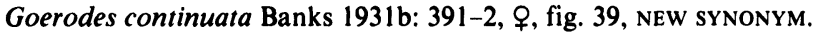

Goerodes continuatus, Fischer 1970: 19.

Goerodes venularis, Fischer 1970: 25.

Male. Color brown. Scape $1.0 \mathrm{~mm}$ long, not highly modified. Maxillary palps $0.6 \mathrm{~mm}$ long. Fore wing (Fig. 21) $7.0-7.5 \mathrm{~mm}$ long. Genitalia (Fig. 22).

Female. Scape $1.0 \mathrm{~mm}$ long, cylindrical. Fore wing $7.5 \mathrm{~mm}$ long. Genitalia (Fig. 23). Spermatheca with two chambers: main chamber with microtrichia sparse, auxiliary chamber smaller, with microtrichia dense, stout, in reticulate pattern.

Material Examined. MAlaysia, PaHANG: Lectotype ô, "Type 16474," "Goerinella venularis type," Cameron's Highlands, $4800 \mathrm{ft}$, 21 VI 1923, HMP, (MCZ); 1 đo syntype, same place, but Tahan, $4500 \mathrm{ft}, 12$ I 1924, MRH, (MCZ); same place as lectotype, 1 ¡े, $6 \mathrm{VI}$ 1935, HMP, (MCZ); same place, 1 ô, 6 VII 1935, HMP, (MCZ); same place, but 45-5000 ft, 1 ㅇ, 14 VI 1935, HMP, (MCZ); same place, but 45-4800 ft, 3 o, 16 VI 1935, HMP, (MCZ); same place, $4700 \mathrm{ft}, 2$ ô, 12 V 1939, HMP, (MCZ); Ginting Kial, $5200 \mathrm{ft}, 1$ ô, 25 VI 1938, HMP, (MCZ). Lectotype 우, "Type 16472," "Goerodes continuata type," Cameron's Highlands, Tahan, $4500 \mathrm{ft}, 12$ I 1924, MRH, (MCZ); 4 \& syntypes: same place, but $4800 \mathrm{ft}, 1$ \&, 12 X 1923; 1 ᄋ, 13 X 1923; 1 \% 12, III 1924, HMP, (MCZ); Sungai Ranglat, 3-500 ft, 1 \&, 10 III 1925, HMP, (MCZ). INDONESIA, SUMATRA: 
Brastagi, 1200 m, 1 ㅇ, 11 XII 1969; 1 ô, 26 XII 1971, (HM); Bukit Tinggi, 9 ㅇ, 24 VIII 1974, (HM); Dairi, 1600 m, 2 đิ, 3 ㅇ, 30 VIII 1970; 4 ô, 3 우, 2 IX 1970; 8 ô, 13 우, 8 XI 1970; 1 ô, 27 XII 1970; 8 ठิ, 25 IV 1971; 6 ठิ, 6 V 1977; 1 ठૈ, 1 ㅇ, 28 VIII 1979, (JSW); Dolok Merangir, 1 \%, 27-31 XII 1969; 1 \%, 1-22 VI 1970; $2 \hat{\delta}, 20$ VI-17 VII 1971; 3 ô, 1 우, 24 III-22 IV 1972; 1 ô, 3 II-19 III 1972; 1 ô, 20 XI 72-5 I 1973; 1 ㅇ, 10 I-3 III 1973; 1 ô, 1 ㅇ, 7 VI-6 X 1974; 1 ô, 3 ㅇ, 22 XII 73-27 I 74; 1 ô, 21 VIII 1976; 1 ô, VI 1981, (HM); Mt. Kerinei, 1800 m, 2 ô, 1 \&, 21-24 II 1976, (HM); Mts. Daior, 5 ô, 6 ㅇ, 17 VIII 1977, (HM); Paritokoa, 1 ㅇ, 22 II 1981, (HM); Prapat,

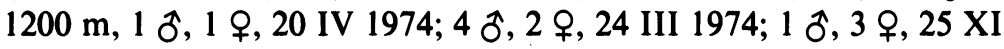
1973, (HM); Sitahoan, 1400 m, 1 ô, 7 ㅇ, 2 IX 1981, (HM); Tele, $1400 \mathrm{~m}, 6$ ठิ, 7 ㅇ, 4 VI 1973, (HM).

\section{Dinarthropsis picea Ulmer}

(Figs. 24, 25)

Dinarthropsis picea Ulmer 1913: 91-2, ठํ क, figs. 9, 10, 12, 13, 15.

Dinarthrodes niger Banks 1913: 142-3, đૈ, pls, 8-9 figs. 4, 5, 13, NEW SYNONYM.

Dinarthropsis picea, Ulmer 1951: 10, 14, 22, 25, 491-4, đิ̊ + , figs. 773-89.

Dinarthropsis nigra, Fischer 1970: 4.

Dinarthropsis picea, Fischer 1970: 4-5.

Male. Color brown. Scape $1.5 \mathrm{~mm}$ long, with two mesal lobes and modified setae. Maxillary palps curved in front of frons. Wings (Fig. 24); fore wing $8.7 \mathrm{~mm}$ long; hind wing with discal cell open. Genitalia (Fig. 25).

Female. Cf. (Ulmer 1913, 1951).

Material Examined. Lectotype đ̄, "Dinarthrodes niger type," "Type 11780," JAVA, no date, Batavia, (MCZ); same data as lectotype, $2 \hat{\delta},(\mathrm{MCZ})$.

\section{Dinarthrodes albicorne (Banks) new comb.}

(Fig. 26)

Crunoecia albicornis Banks 1906: 109,, pl. 3 fig. 11.

Crunoecia albicornis, Fischer 1970: 71.

The hind wing of this species has a closed discal cell and thus, it should be removed from Crunoecia. It is tentatively placed in the genus Dinarthrodes, because in the female the scape is relatively long and the wings are not broad.

Male. Unknown. 
Female. Color dark brown. Scape $2.0 \mathrm{~mm}$ long. Fore wing 9.0 $\mathrm{mm}$ long. Hind wing with open discal cell. Genitalia (Fig. 26).

Material Examined. Lectotype \%, "Type 11830," JAPAN, Hikosan, Buzen, no date, (MCZ); same data as lectotype, 1 \%, (MCZ).

\section{GOERIDAE}

Goera mustellina (Hagen) new comb.

Mormonia mustellina Hagen 1859: 209.

Goerodes mustellinus, Fischer 1970: 22.

I have not examined any syntypes of this species. However, Oliver $\mathrm{S}$. Flint has informed me that it is another species of Goera.

\section{ACKNOWLEDGMENTS}

This study would not have been possible without the assistance of the following people for providing lepidostomatid material to me from their institutions: Peter C. Barnard, British Museum; Oliver S. Flint, Jr., Smithsonian Institution; Hans Malicky, Biological Station Lunz; and Alfred F. Newton, Jr., Museum of Comparative Zoology. Also, my thanks to Donald S. Chandler and R. Marcel Reeves, University of New Hampshire, for reviewing the manuscript.

\section{SUMMARY}

The Oriental Lepidostomatidae described by Banks and Hagen are redescribed and lectotypes are designated, including: Goerodes abruptus Banks, G. apoanus (Banks), G. bicolor (Banks), G. conjunctus (Banks), G. grandis (Banks), G. medius (Banks), G. minor (Banks), G. piscinus (Hagen), G. posticatus (Banks), G. ursinus (Hagen) G. venularis (Banks). Two new combinations: Goera mustellina (Hagen), removed from Goerodes: and Dinarthrodes albicorne (Banks), removed from Crunoecia. Three new synonyms: Goerodes continuatus Banks, junior synonym of $G$. venularis (Banks); G. punda Mosely, a junior synonym of $G$. ursinus (Hagen); and Dinarthropsis nigra (Banks) a junior synonym of $D$. picea Ulmer. 


\section{Literature Cited}

BANKS, N.

1906 (1905). New Trichoptera from Japan. Proc. Entomol. Soc. Wash., 7: 106-13.

1913. New exotic neuropteroid insects. Proc. Entomol. Soc. Wash., 15: 137-43.

1931a. Some Oriental neuropteroid insects. Psyche, 38: 56-70, 1 pl.

1931b. Neuropteroid insects from the Malay Peninsula. J. Fed. Malay States Mus., 16: 377-410, 3 pls.

1931c. Neuropteroid insects from North Borneo, particularly from Mt. Kinabalu. J. Fed. Malay States Mus., 16: 411-30, 2 pls.

1934. Supplementary neuropteroid insects from Mt. Kinabalu, Borneo, J. Fed. Malay States Mus., 17: 567-78.

1937. Philippine neuropteroid insects. Philipp. J. Sci., 63: 125-74, 6 pls.

FisCHER, F. C. J.

1970. Trichopterum Catalogus. Amsterdam, Nederlandse Entomol. Vereeninging, 11: vi $+316 \mathrm{p}$.

HAGEN, H. A.

1858. Synopsis der Neuroptera Ceylons. Pars I. Verh. Zool. Bot. Ges. Wien, 8: 471-88.

1859. Synopsis der Neuroptera Ceylons. Pars II. Verh. Zool. Bot. Ges. Wien, 9: 208-12.

Ross, H. H.

1938. Lectotypes of North American caddis flies in the Museum of Comparative Zoology. Psyche, 45: 1-61, 10 pls.

Mosely, M. E.

1949. The Indian caddis flies (Trichoptera). Part XI. J. Bombay Nat. Hist. Soc., 48: 782-91, 12 pls.

ULMER, G.

1913. Uber einige von Edw. Jacobson auf Java Gesammelte Trichopteren. Zweiter Beitrag. Notes Leyden Mus., 35: 78-101.

1951. Köcherfligen (Trichoptera) von den Sunda-Inseln. Tiel I. Arch. Hydrobiol. Suppl. 19: 1-528. 

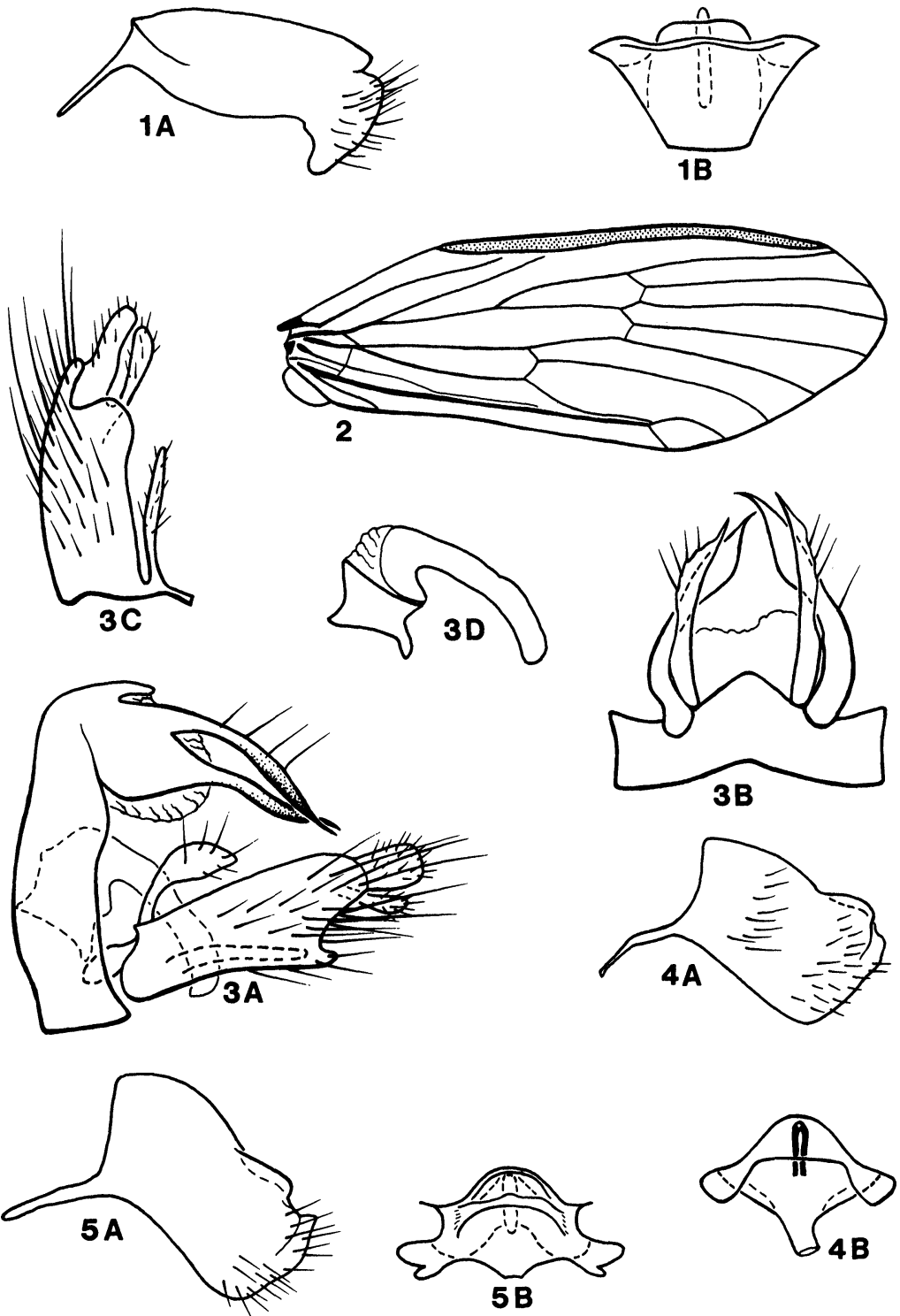

Figs. 1-5. 1. Goerodes abruptus, $ᄋ$ genitalia, A. segment IX, lateral; B. spermathecal sclerite, ventral. 2-4. G. apoanus: 2 . $\hat{\delta}$ fore wing. 3. $\hat{\delta}$ genitalia, A. lateral; B. segments IX and X, dorsal; $C$. inferior appendage, ventral; D. phallus, lateral. 4. \& genitalia, A. segment IX, lateral; B. spermathecal sclerite, ventral. 5. G. bicolor, $\$$ genitalia, A. segment IX, lateral; B. spermathecal sclerite, ventral. 

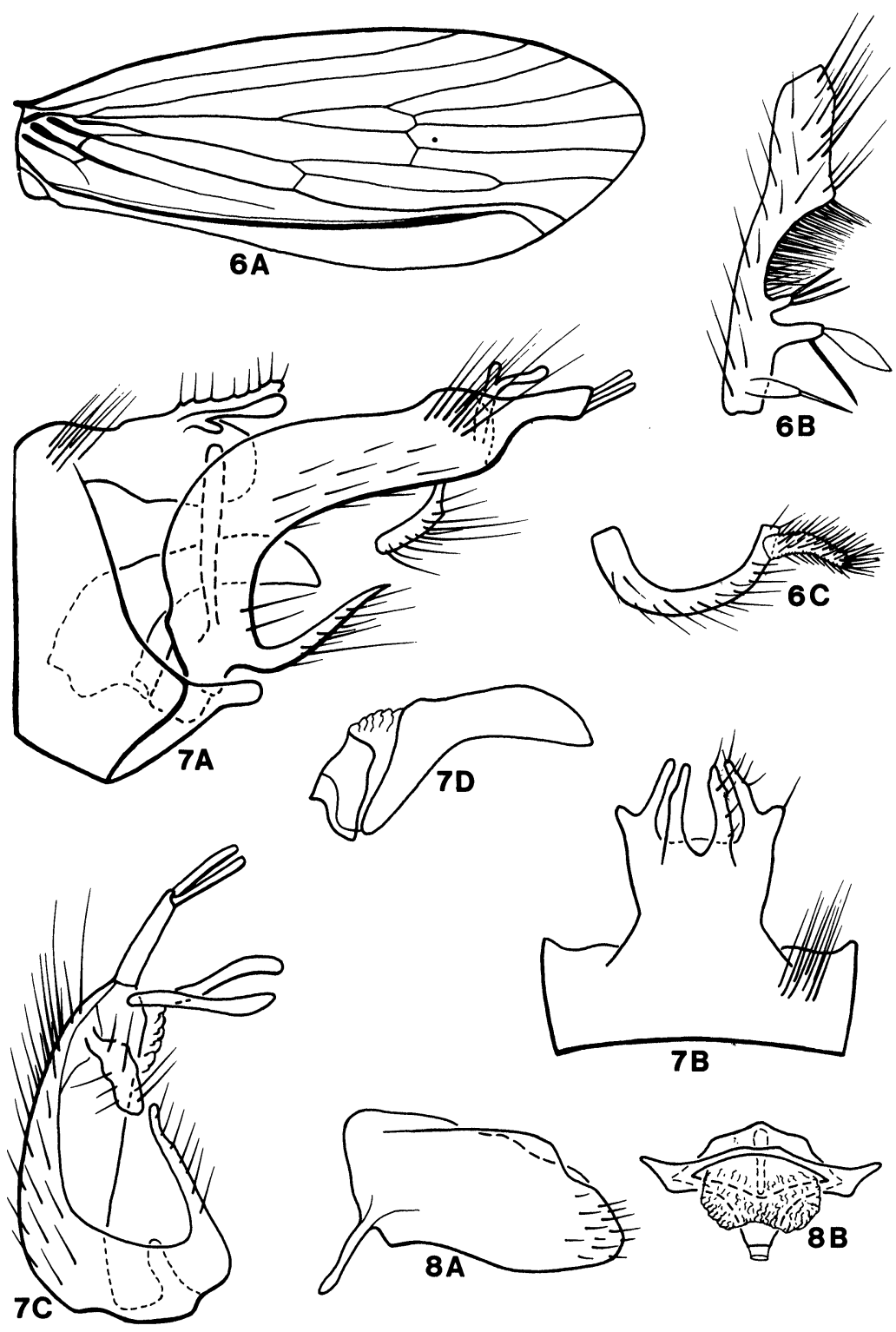

Figs. 6-8 Goerodes conjunctus: 6. $\hat{\delta}$, A. fore wing; B. scape, left, dorsal; C. maxillary palp, left, mesal. 7. ô genitalia, A. lateral; B. segments IX and X, dorsal; C. inferior appendage, ventral; D. phallus, lateral. 8. $\%$ genitalia, A. segment IX, lateral; B. spermathecal sclerite, ventral. 

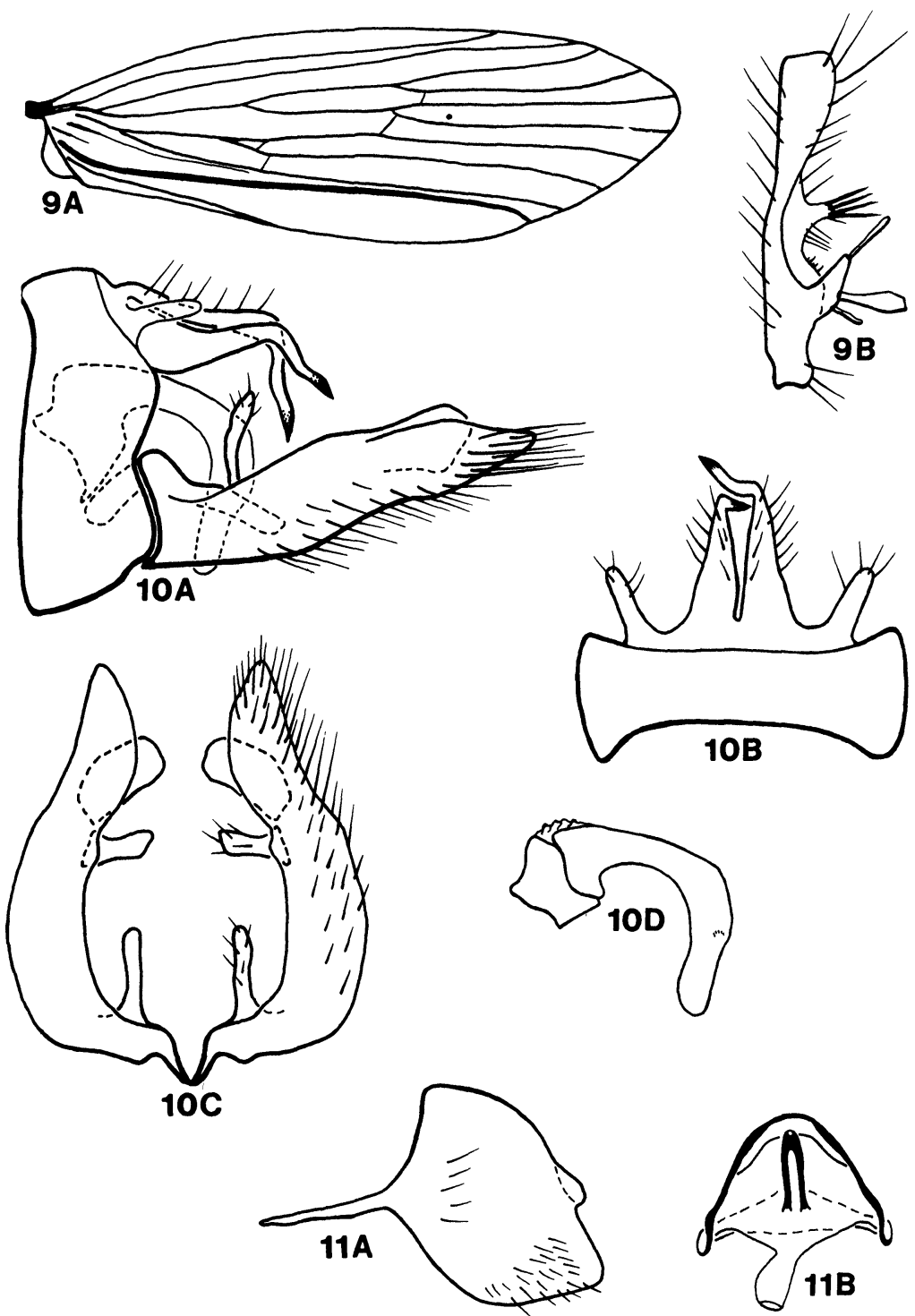

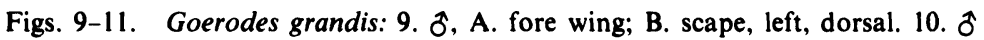
genitalia, A. lateral; B. segments IX and X, dorsal; C. inferior appendages, ventral; D. phallus, lateral. 11. $q$ genitalia, A. segment IX, lateral; B. spermathecal sclerite, ventral. 

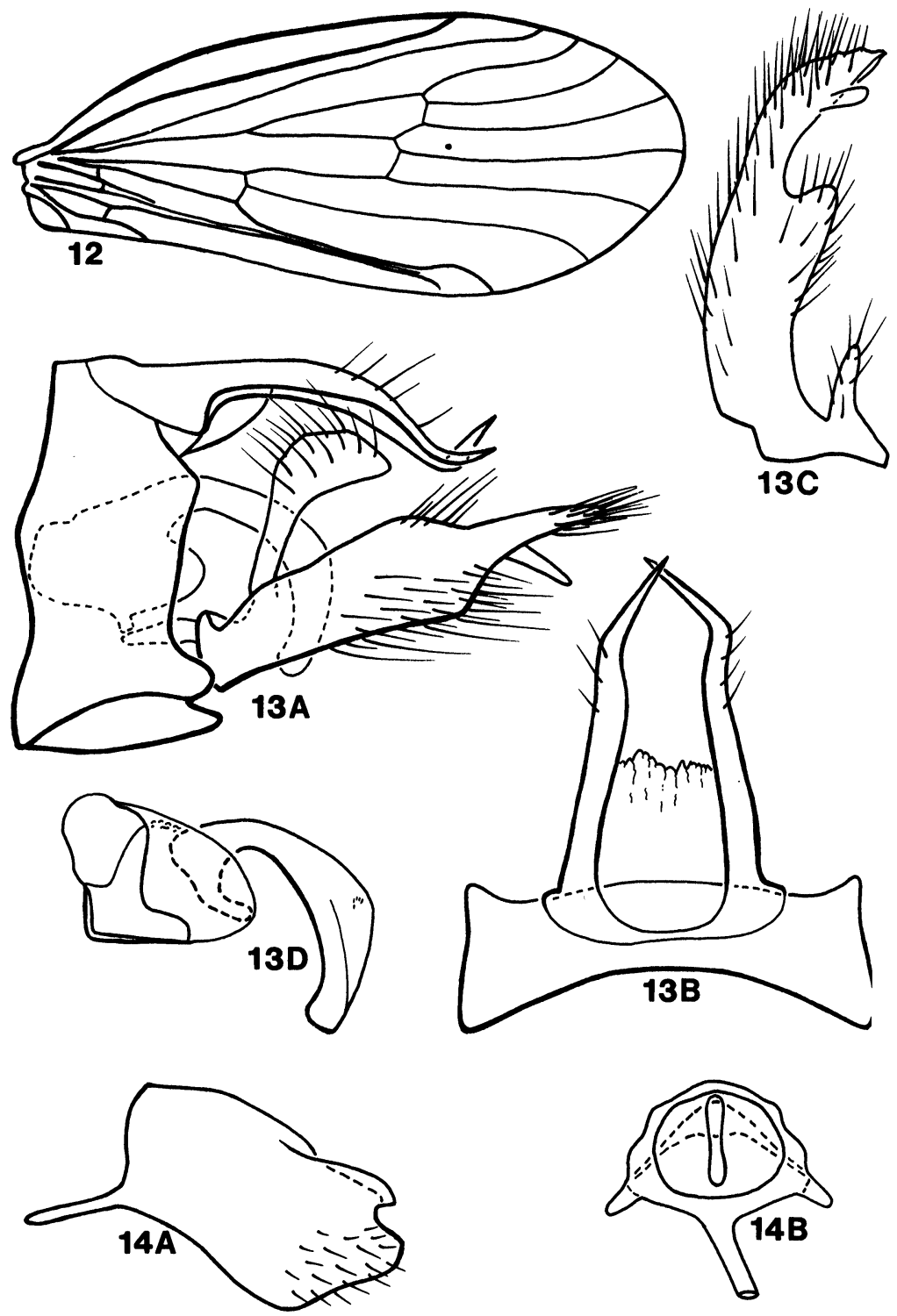

Figs. 12-14. Goerodes medius: 12. $\hat{o}$ fore wing. 13. ô genitalia, A. lateral; B. segments IX and X, dorsal; C. inferior appendage, ventral; D. phallus, lateral. 14. $\%$ genitalia, A. segment IX; B. spermathecal sclerite, ventral. 


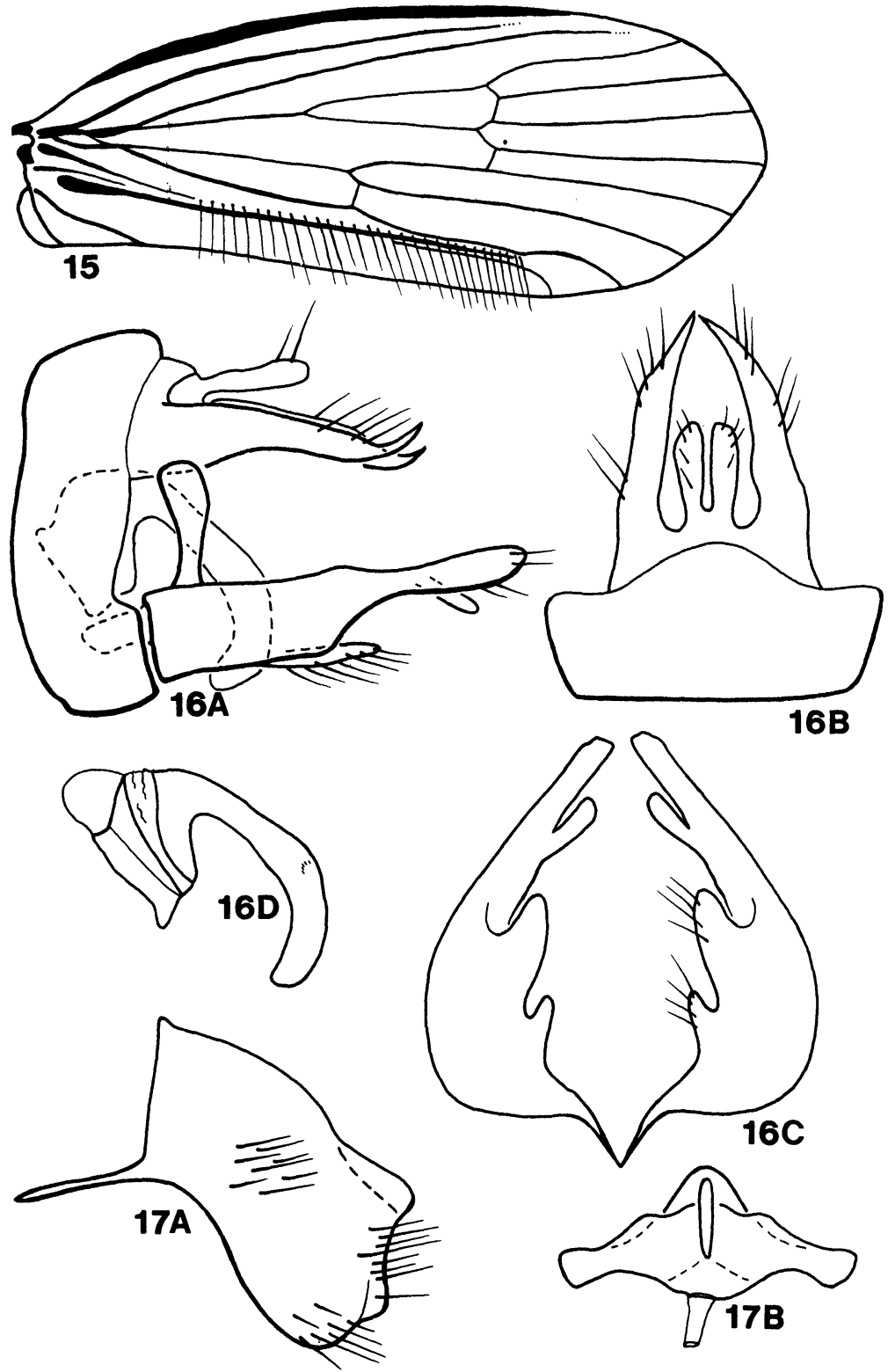

Figs. 15-17. 15-16. Goerodes minor, ठิ: 15. fore wing. 16. genitalia, A. lateral; B. segments IX and X, dorsal; C. inferior appendages, ventral; D. phallus, lateral. 17. G. piscinus, $\&$ genitalia, A. segment IX, lateral; B. spermathecal sclerite, ventral. 

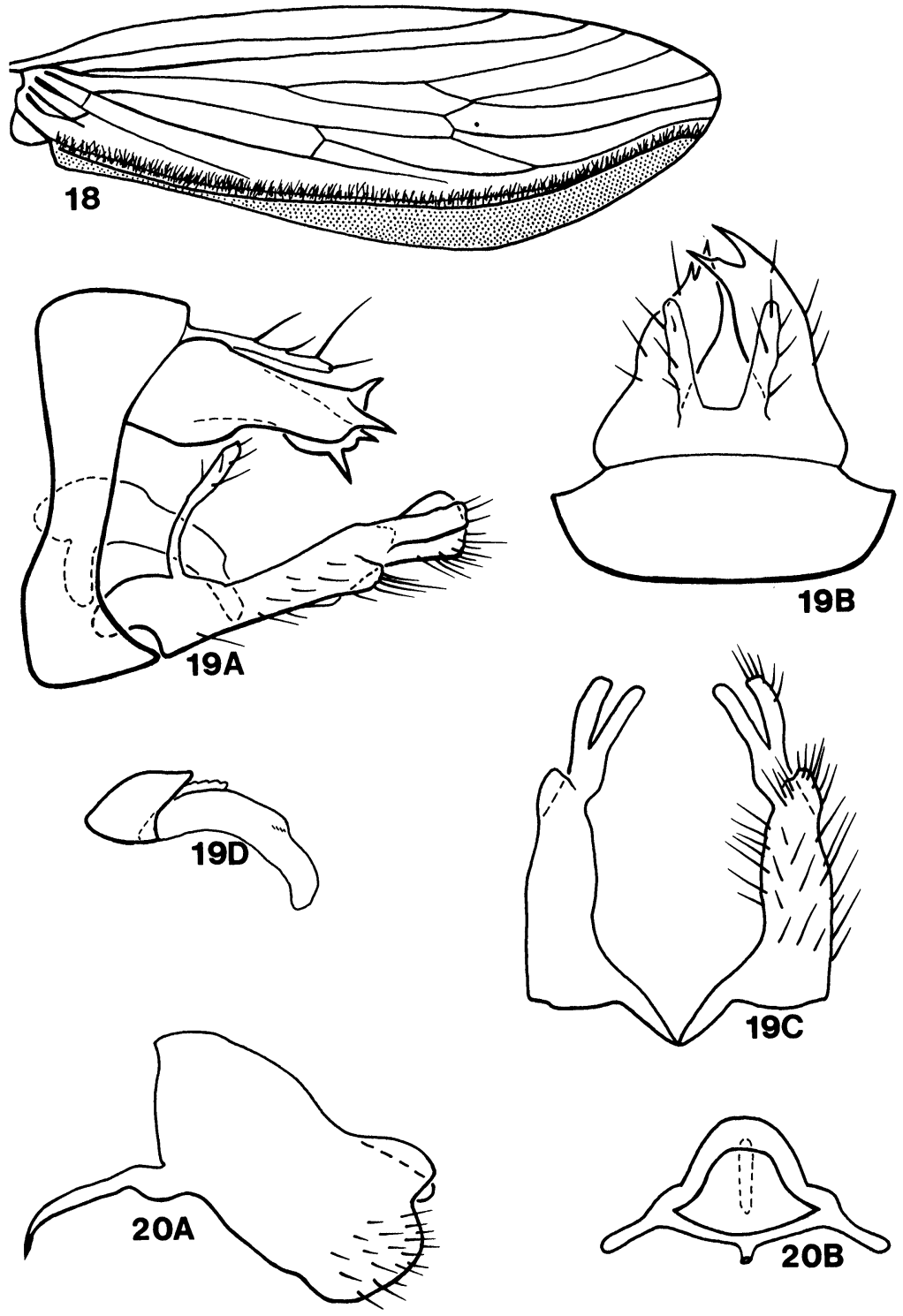

Figs. 18-20. 18-19. Goerodes posticatus, ð̋: 18. fore wing; 19. genitalia, A. lateral; B. segments IX and X, dorsal; C. inferior appendages, ventral; D. phallus, lateral. 20. G. ursinus, 9 genitalia, A. segment IX, lateral; B. spermathecal sclerite, ventral. 

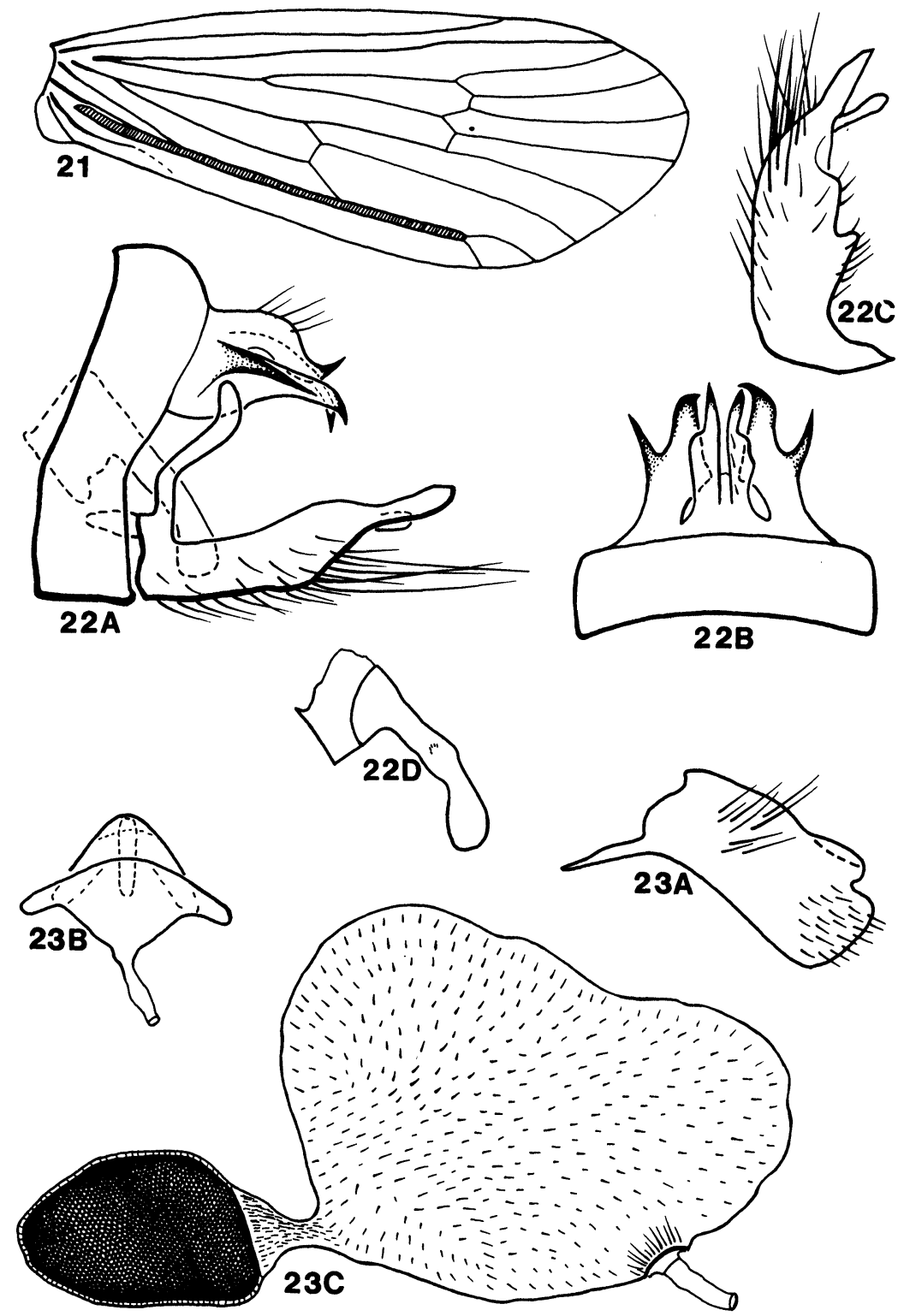

Figs. 21-23. Goerodes venularis: 21. $\widehat{\partial}$ fore wing. 22. $\widehat{\partial}$ genitalia, A. lateral; B. segments IX and X; C. inferior appendage, lateral; D. phallus, lateral. 23. + genitalia, A. segment IX, lateral; B. spermathecal sclerite, ventral; $C$. spermatheca, lateral. 

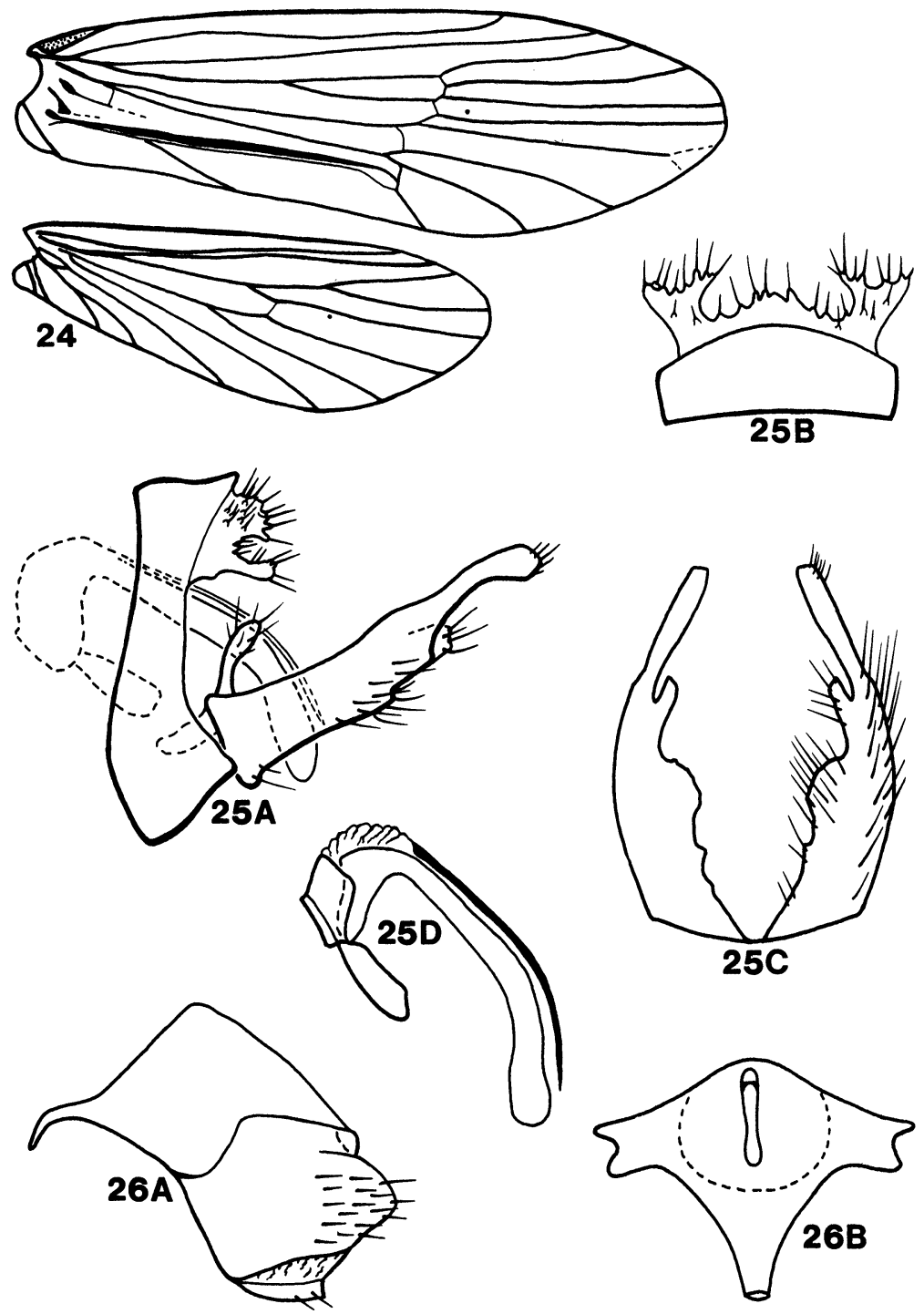

Figs. 24-26. 24-25. Dinarthropsis picea, đ̊: 24. wings. 25. genitalia, A. lateral; B. segments IX and X, dorsal; C. inferior appendages; D. phallus, lateral. 26. Dinarthrodes albicorne, $q$ genitalia, A. segment IX, lateral; B. spermathecal sclerite, ventral. 

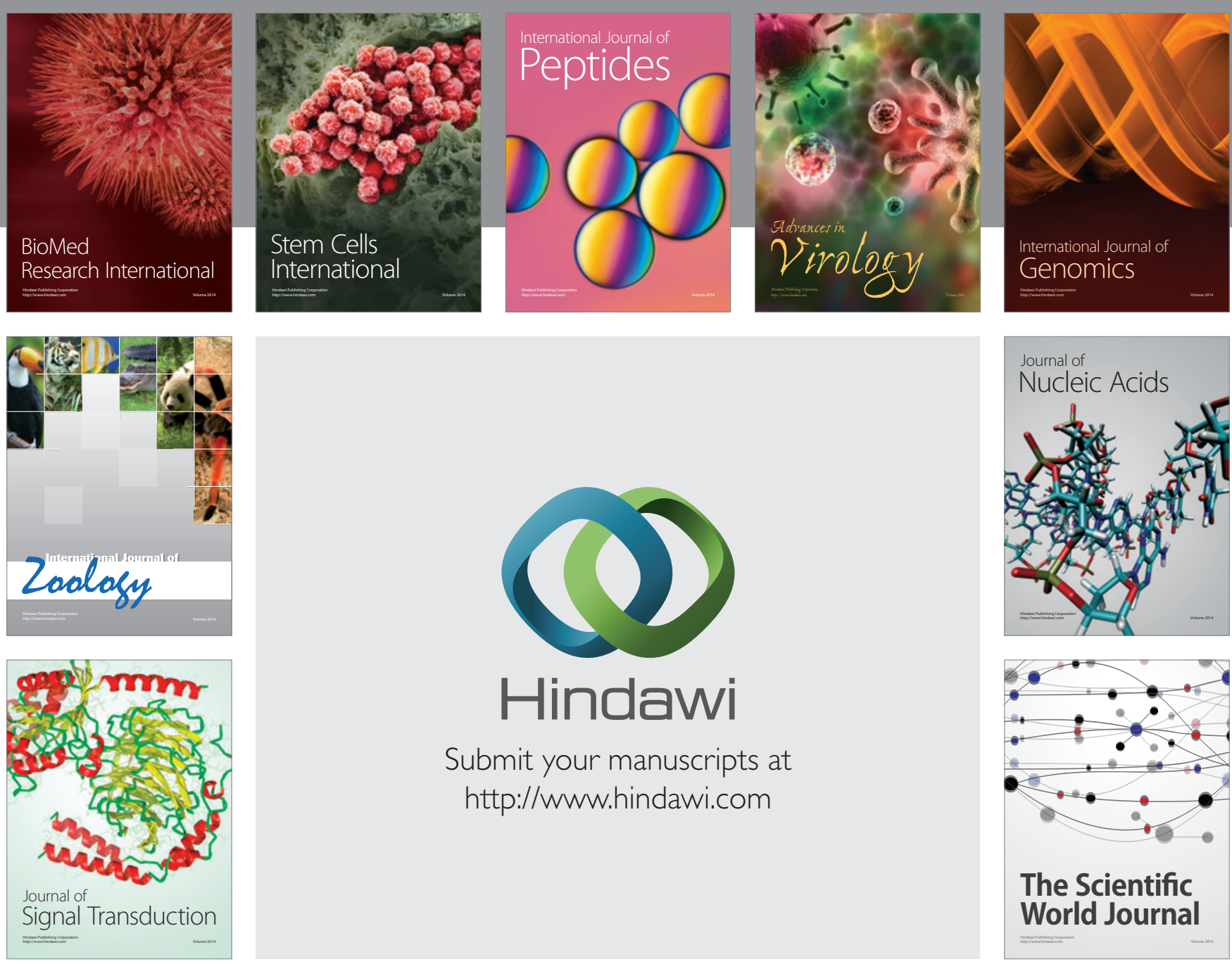

Submit your manuscripts at

http://www.hindawi.com
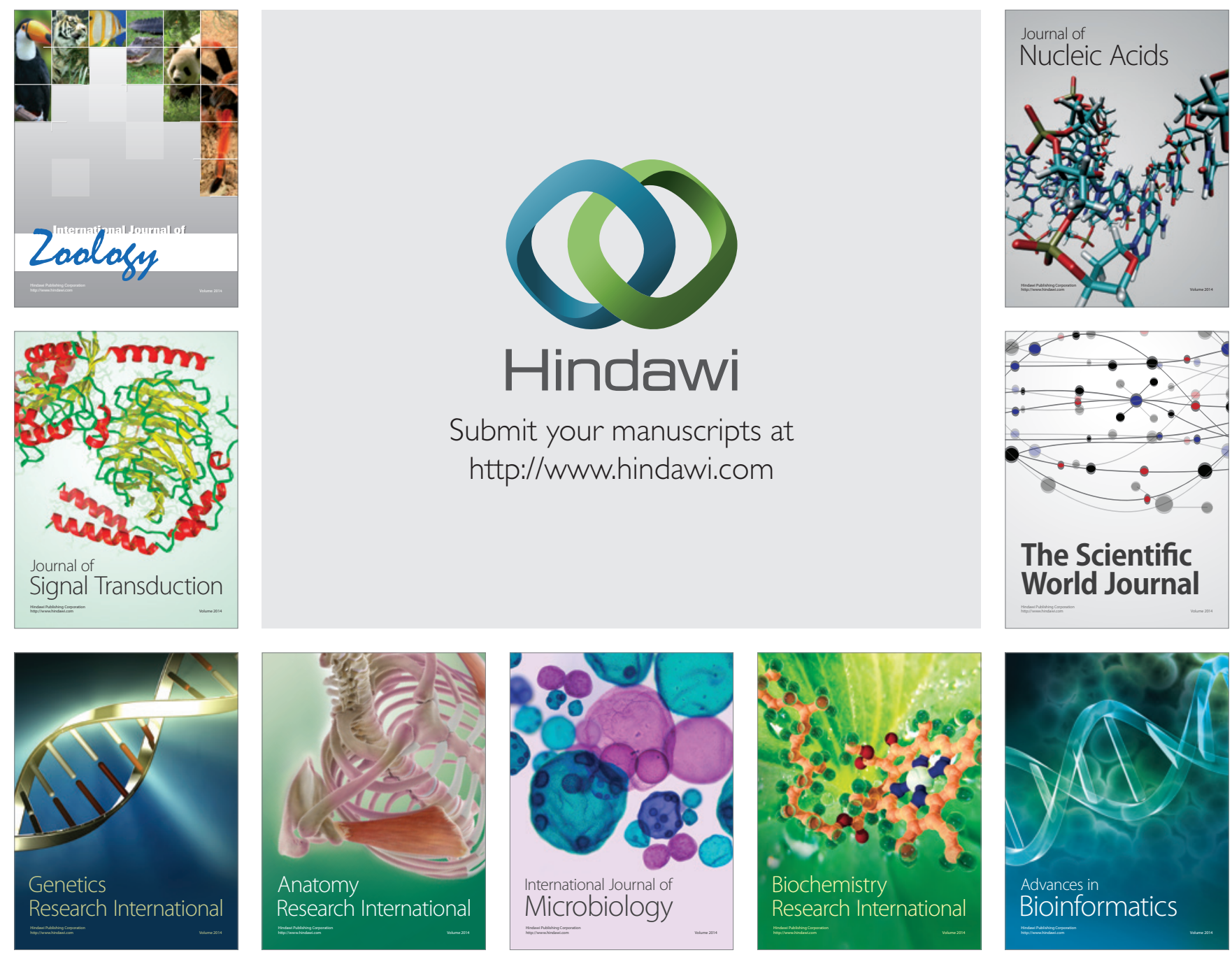

The Scientific World Journal
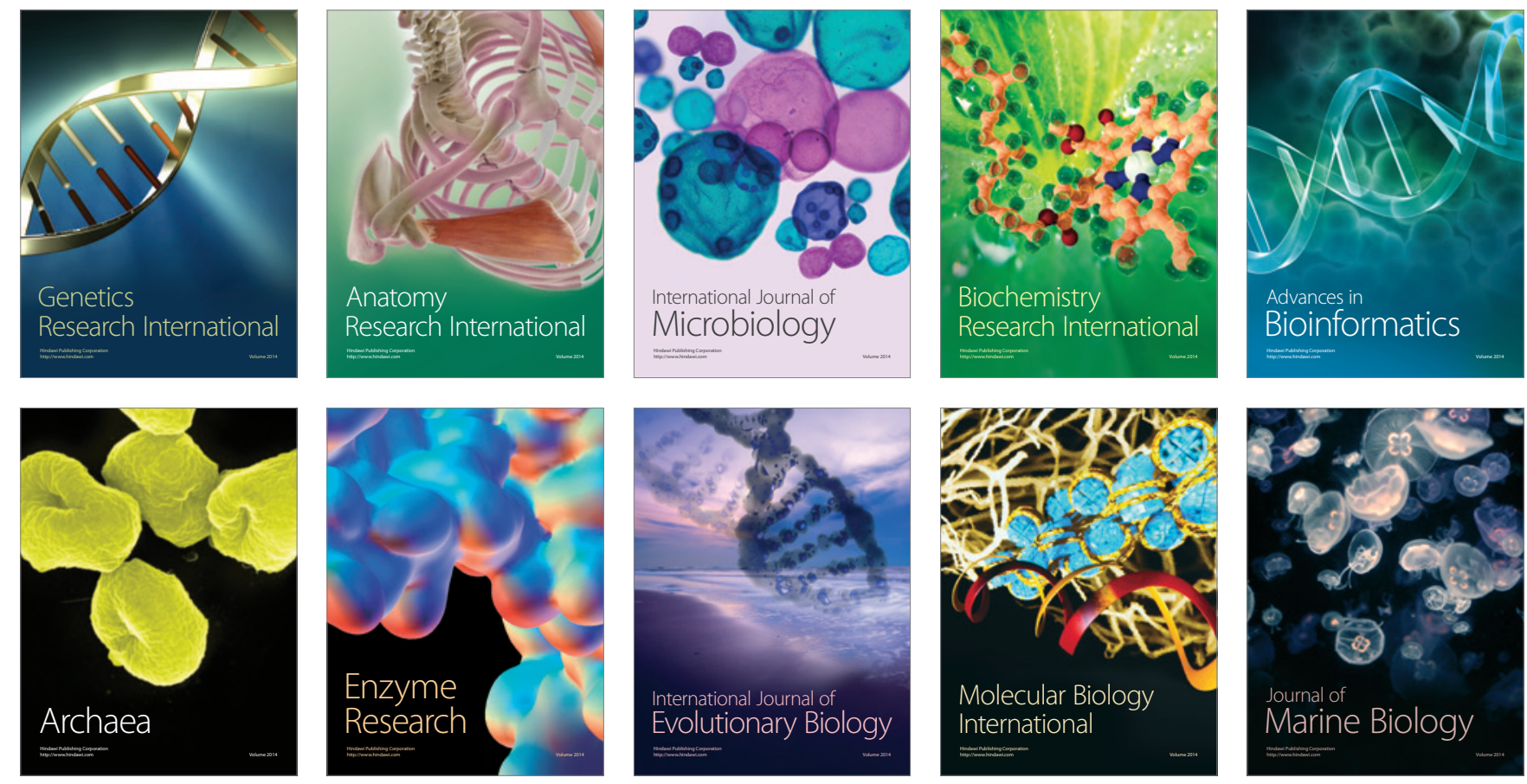